\title{
Surface Impedance Boundary Condition with Circuit Coupling for the 3D Finite Element Modeling of Wireless Power Transfer
}

\author{
A. Desmoort ${ }^{1}$, Z. De Grève ${ }^{1}$, P. Dular ${ }^{2}$, C. Geuzaine ${ }^{2}$, and O. Deblecker ${ }^{1}$ \\ ${ }^{1}$ Electrical Power Engineering Unit, University of Mons, B-7000 Mons, Belgium \\ ${ }^{2}$ Department of Electrical Engineering and Computer Science, ACE, University of Liège, B-4000 Liège, Belgium
}

\begin{abstract}
A light 3D finite element magnetodynamic a- $v$ model of resonant wireless power transfer (WPT) coils using 3D surface impedance boundary condition (SIBC) strongly coupled with an external circuit is proposed, reflecting the importance of external circuit elements (notably capacitances) in the resonance phenomena at circuit and field levels. The computational gain ensuing from the use of SIBC instead of massive conductor formulations is demonstrated on an academic example. The method is validated by comparing the simulated and experimentally measured input impedance of a complete resonant WPT system, attesting the correct behavior of the model while saving important computational resources.
\end{abstract}

Index Terms-Finite element, inductive power transfer, surface impedance boundary condition

\section{INTRODUCTION}

$\mathbf{T}$ HE wireless power transfer (WPT) is a trending topic. Indeed, the recent progresses in power electronics have paved the way to the implementation of resonant inductive power transfer for energy-greedy applications, such as the supply of electric vehicles [1]. Bringing significative information, the 3D finite element (FE) modeling of the coils used for resonant WPT as massive conductors is nevertheless submitted to important computational burden. The skin and proximity effects appearing at the working frequency level (tens of $\mathrm{kHz}$ for the transfer of high power) require a high refinement of the conductors volume mesh, making massive conductors formulations hardly applicable. The resorts to stranded conductors [2] or homogenization technique [3] have therefore been investigated. Here, profit is taken from a 3D surface impedance boundary condition (SIBC) [4]-[5] to avoid the volume mesh inside the conductors and relax the computational constraints without using homogenization. Reflecting the important influence of the external circuit on the power transfer (through the resonant effect), a circuit coupling needs to be applied in order to model the resonant conditions at the circuit and field levels. The combination of SIBC with a circuit-coupled $\mathbf{t}-\phi$ formulation (with $\mathbf{t}$ the electric vector potential and $\phi$ the magnetic scalar potential) has already been addressed in [6]. Here, an $\mathbf{a}-v$ formulation (with a the magnetic vector potential and $v$ the electric scalar potential) is considered. A natural strong circuit coupling method for $\mathbf{a}-v$ formulation involving massive conductors has been proposed in [7]. In this work, this last contribution is adapted and extended to the use of SIBC, leading to a new way to implement SIBC with circuit coupling.

Manuscript received November 20, 2016; revised January 7, 2017 and January 29, 2017; accepted January 29, 2017. Date of publication July 10, 2015; date of current version July 31, 2015. Corresponding author: A. Desmoort (e-mail: alexis.desmoort@umons.ac.be).

Color versions of one or more of the figures in this paper are available online at http://ieeexplore.ieee.org.

Digital Object Identifier (inserted by IEEE).
In section II, the magnetodynamic a- $v$ weak formulation is reminded, while the concept of SIBC and its introduction in the $\mathbf{a}-v$ weak form are addressed. Section III presents the methodology for implementing a strong circuit coupling in $\mathbf{a}-v$ magnetodynamics involving SIBC, which is the main contribution of this work. In section IV, the computational gain afforded by the use of SIBC is highlighted on an academic example. Finally, in section V, the method is validated by the comparison of simulation and experimental results.

\section{A- $v$ FORMULATION WITH SIBC}

\section{A. Magnetodynamic problem and $\boldsymbol{a}-v$ weak formulation}

A magnetodynamic problem is defined on a bounded domain $\Omega$ of the Euclidian space, including conducting parts $\Omega_{\mathrm{c}}$. By resorting to the magnetic vector potential $\mathbf{a}$ and to the electric scalar potential $v$, the problem consists in finding a $\in \mathrm{F}_{\mathbf{a}}^{1}(\Omega)$, of which uniqueness is ensured in this work by a gauge condition presented in [8], and $v \in \mathrm{F}_{v}^{0}(\Omega)$, so that [9]:

$$
\begin{aligned}
\left(\mu^{-1} \operatorname{curl} \mathbf{a}, \operatorname{curl} \mathbf{a}^{\prime}\right)_{\Omega}+\left\langle\mathbf{n} \times \mathbf{h}, \mathbf{a}^{\prime}\right\rangle_{\Gamma_{\mathrm{h}}} & \\
+\left(\sigma \partial_{t} \mathbf{a}, \mathbf{a}^{\prime}\right)_{\Omega_{c}}+\left(\sigma \operatorname{grad} v, \mathbf{a}^{\prime}\right)_{\Omega_{c}} & =0, \\
& \forall \mathbf{a}, \in \mathrm{F}_{\mathbf{a}}^{1}(\Omega)
\end{aligned}
$$

and

$$
\begin{aligned}
\left\langle\mathbf{n} \times \mathbf{h}, \operatorname{grad} v^{\prime}\right\rangle_{\Gamma_{\mathrm{h}}}+\left(\sigma \partial_{t} \mathbf{a}, \operatorname{grad} v^{\prime}\right. & \\
+\left(\sigma \operatorname{grad} v, \operatorname{grad} v^{\prime}\right)_{\Omega_{c}} & =0, \\
& \forall v^{\prime} \in \mathrm{F}_{v}^{0}(\Omega)
\end{aligned}
$$

where $\mu$ is the magnetic permeability, $\mathbf{n}$ is the unit normal vector exterior to $\Omega, \mathbf{h}$ is the magnetic field, $\Gamma_{\mathrm{h}}$ is the part of the boundary of $\Omega$ concerned by Neumann's or mixed boundary conditions and $\sigma$ is the electric conductivity. $\mathrm{F}_{\mathbf{a}}^{1}(\Omega)$ and $\mathrm{F}_{v}^{0}(\Omega)$ are, respectively, curl- and grad-conform function spaces containing, respectively, the basis functions of $\mathbf{a}$ and of $v .(., .)_{\Omega}$ and $\langle., .\rangle_{\Gamma}$ denote respectively a volume integral in $\Omega$ and a surface integral on $\Gamma$ of the product of their arguments. Solving (1) and (2) is similar to respectively solve curl $\mathbf{h}=\mathbf{j}$ in $\Omega$ and $\operatorname{div} \mathbf{j}=0$ in $\Omega_{\mathrm{c}}$ in a weak sense. 


\section{B. Surface impedance boundary condition}

The conducting parts $\Omega_{c}$ can be removed from $\Omega$ while keeping the eddy currents effects into the problem. Indeed, following from Snell's law of refraction, the law relating the tangential traces of the magnetic and electric fields on the dielectric/conductor interface can be established. This relation is assumed to be constant over the conductor's surface (it is independent from the position) and may thus act as a particular local boundary condition applied on the conducting parts $\Gamma_{\mathrm{c}}$ of boundary $\Gamma_{\mathrm{h}}$. Assuming that the electromagnetic fields are penetrating the conductor normally to its surface, their tangential traces are related by the following equation:

$$
\mathbf{n} \times\left.\mathbf{h}\right|_{\Gamma_{\mathrm{c}}}=Z_{\mathrm{c}}^{-1} \mathbf{n} \times\left.(\mathbf{n} \times \mathbf{e})\right|_{\Gamma_{\mathrm{c}}}
$$

where $\mathbf{e}$ is the electric field. It can be intuitively understood that the norms of the fields are linked through a coefficient pertaining to an impedance at each point of the interface surface, i.e., the conductor local surface impedance $Z_{c}$. This particular type of local boundary condition is thus called SIBC. In the frequency domain, the surface impedance is approached by an asymptotic expansion with respect to the skin depth $\delta$ [4]-[5]. In this work, the frequency level is expected to be high enough to neglect higher orders than the first one since the skin depth is low enough in comparison with conductors dimension, which is confirmed by the selection criteria presented in [10]. In this case and assuming $\sigma \gg \omega \varepsilon$ in the conductors (with $\varepsilon$ the electric permittivity and $\omega$ the angular frequency), the expression of the surface impedance is limited to:

$$
Z_{\mathrm{c}} \approx \frac{\mu \omega}{2}(1+j) \delta=\frac{(1+j)}{\sigma \delta}
$$

where $j$ is the imaginary unit (so that $\partial_{t} \equiv j \omega$ ). Equation (4) is known as the Leontovich approximation and is rigorously exact in the case of an infinite conducting plane (presenting a finite conductivity). As additional information, it can be noted that the second order approximation (i.e., the Mitzner approximation) takes a possible curvature of the conducting plane into account whereas the third order approximation (i.e., the Rytov approximation) allows the inclusion of the fields tangential diffusion [4]-[5]. The SIBC expressed in terms of the potentials a and $v$ is introduced in the weak forms (1) and (2) through the boundary term, as $\Gamma_{\mathrm{c}} \subset \Gamma_{\mathrm{h}}$, with:

$$
\begin{aligned}
\left\langle\mathbf{n} \times \mathbf{h}, \mathbf{e}^{\prime}\right\rangle_{\Gamma_{\mathrm{c}}} & =\left\langle Z_{\mathrm{c}}^{-1}\left(\mathbf{n} \times\left(\partial_{t} \mathbf{a}+\operatorname{grad} v\right)\right) \times \mathbf{n}, \mathbf{e}^{\prime}\right\rangle_{\Gamma_{\mathrm{c}}} \\
& =\left\langle Z_{\mathrm{c}}^{-1} \mathbf{n} \times\left(\partial_{t} \mathbf{a}+\operatorname{grad} v\right), \mathbf{n} \times \mathbf{e}^{\prime}\right\rangle_{\Gamma_{\mathrm{c}}}
\end{aligned}
$$

where e' is whether a' or grad $v^{\prime}$. The conductors are now represented by their tubular skin only, and $v$ is now only defined on and supported by the boundary $\Gamma_{c}$.

\section{CiRcuit COUPLING}

The methodology to achieve a strong circuit coupling consists in adding constraints involving global quantities (i.e., the voltage and the current of the conductors) to the SIBC magnetodynamic formulation (1)-(2)-(5). This approach extends the methodology presented in [7], which focuses on massive conductors only, to the use of SIBC.

\section{A. Defining constraints involving global quantities}

The coupling of the problem with an external circuit requires to define constraints involving global quantities. It can be achieved by locating an electromotive force between two infinitely close cross-sections (representing the electrodes) of each inductor $i$, with $i \in \mathcal{C}$ (see Fig. 1). As the conducting parts have been removed from the domain, the persisting parts of the electrodes are their contour exclusively. The thin tube separating those contours is denoted $\Gamma_{\mathrm{g}}^{i}$ and extracted from $\Omega$. Let $V_{i}$ and $I_{i}$ be respectively the voltage between the electrodes and the current across one of the electrode, whose contour is denoted $\gamma_{\mathrm{g}}^{i}$. One has:

$$
\int_{\gamma_{i}} \mathbf{e} \cdot \mathrm{d} \mathbf{l}=V_{i} \text { and } \int_{\gamma_{\mathrm{g}}^{i}} \mathbf{h} \cdot \mathrm{d} \mathbf{l}=I_{i}
$$

where $\gamma_{i}$ is any path in $\Gamma_{\mathrm{g}}^{i}$ connecting the electrodes. In the next subsections, the introduction of global quantities constraints is implemented in a- $v$ magnetodynamics with SIBC.

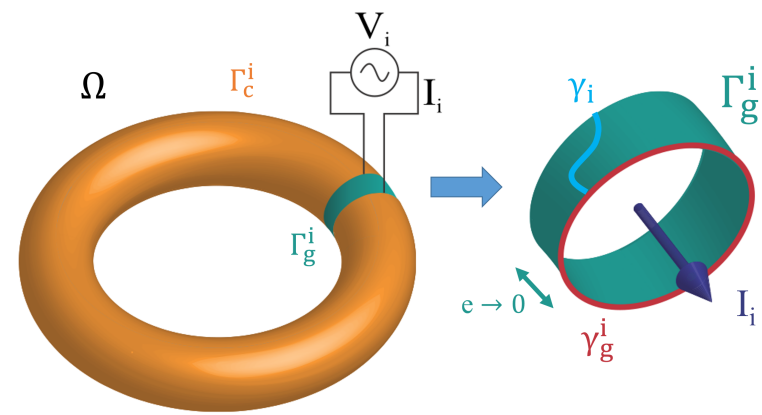

Fig. 1: Electromotive force definition in the inductor $i$

\section{B. Voltage as a strong global quantity}

Until this point, the concepts have been established in general terms, at the continuous level. Nevertheless, the transition to the discrete level is mandatory. If edge and nodal elements are respectively used for $\mathbf{a}$ and $v$, the gradient of $v^{\prime}$ is included in the space of a' [11] so that the weak form (2) of $\operatorname{div} \mathbf{j}=0$ is included in the weak form (1) of curl $\mathbf{h}$ $=\mathbf{j}$. As a consequence, the problem can be segmented into two successive subproblems. The first subproblem consists in determining the source surface electric scalar potentials $v_{0, s}^{i}$, defined on $\Gamma_{\mathrm{c}}^{i}$, corresponding to the application of a unit voltage to each conductor $i$. This can be done by solving electrokinetics problems for instance. Then, $v$ is expressed as

$$
v=\sum_{i \in \mathcal{C}} V_{i} v_{0, s}^{i} .
$$

However, in order to avoid the resort to another formulation, a generalized source surface scalar potential can be defined as the sum of the nodal basis functions of all the nodes located on the contour $\gamma_{\mathrm{g}}^{i}$ modeling one of the electrode. As shown on Fig. 2, its support is thus limited to a transition layer made of the surface elements on $\Gamma_{\mathrm{c}}^{i}$ and adjacent to $\gamma_{\mathrm{g}}^{i}$. It is then equal to 1 on $\gamma_{\mathrm{g}}^{i}$ and decreases to 0 in the transition layer. This reinterpretation appears to be an optimal choice as it avoids a pre-computation and yields an improved band width in the system of equations as discussed in [7]. 


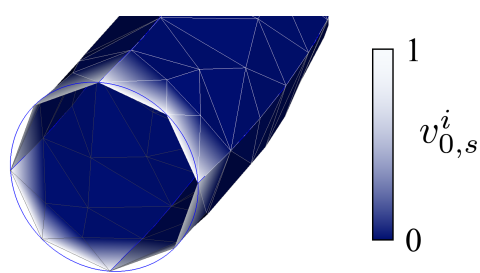

Fig. 2: Generalized source surface scalar potential $v_{0, s}^{i}$

The second subproblem consists in solving the weak form (1) where the voltages $V_{i}$ have replaced the potential $v$ as unknowns using (7). Therefore, the voltage has been introduced as a strong quantity in the problem, which leads to, after some vector algebra operations:

$$
\begin{aligned}
& \left(\mu^{-1} \operatorname{curl} \mathbf{a}, \operatorname{curl} \mathbf{a}^{\prime}\right)_{\Omega}+\left\langle\mathbf{n} \times \mathbf{h}, \mathbf{a}^{\prime}\right\rangle_{\Gamma_{\mathrm{h}} \backslash \Gamma_{\mathrm{c}}} \\
& +\left\langle Z_{\mathrm{c}}^{-1} \mathbf{n} \times \partial_{t} \mathbf{a}, \mathbf{n} \times \mathbf{a}\right\rangle_{\Gamma_{\mathrm{c}}} \\
& +\sum_{i \in \mathcal{C}} V_{i}\left\langle Z_{\mathrm{c}}^{-1} \mathbf{n} \times \operatorname{grad} v_{0, s}^{i}, \mathbf{n} \times \mathbf{a}^{\prime}\right\rangle_{\Gamma_{\mathrm{c}}^{i}}=0 .
\end{aligned}
$$

Finally, the problem needs to be completed by defining as many circuit relations (involving the current $I_{i}$ ) as there are inductors in the problem.

\section{Current as a weak global quantity and circuit relations}

The current flowing into an inductor $i$ is obtained by taking $\mathbf{a}^{\prime}=\operatorname{grad} v_{0, s}^{i}$ as test-function in the weak form (8). Indeed, as curl grad $v_{0, s}^{i}=0$, the volume term is 0 . Then, the boundary term affecting $\Gamma_{\mathrm{h}} \backslash \Gamma_{\mathrm{c}}$ is decomposed into two terms using vector algebra rules:

$$
\begin{aligned}
& \left\langle\mathbf{n} \times \mathbf{h}, \operatorname{grad} v_{0, s}^{i}\right\rangle_{\Gamma_{\mathrm{h}} \backslash \Gamma_{\mathrm{c}}}= \\
& \int_{\Gamma_{\mathrm{h}} \backslash \Gamma_{\mathrm{c}}} \mathbf{n} \cdot \operatorname{curl}\left(\mathbf{h} v_{0, s}^{i}\right) \mathrm{d} \Gamma-\int_{\Gamma_{\mathrm{h}} \backslash \Gamma_{\mathrm{c}}} \mathbf{n} \cdot \operatorname{curl} \mathbf{h} v_{0, s}^{i} \mathrm{~d} \Gamma .
\end{aligned}
$$

The second term is always 0 since the $v_{0, s}^{i}$ are only defined on $\Gamma_{\mathrm{c}}$. The Stokes theorem is applied on the first term, turning the surface integral into a contour integral on the boundaries of $\Gamma_{\mathrm{h}} \backslash \Gamma_{\mathrm{c}}$. As the $\Gamma_{\mathrm{g}}^{k}(k \in \mathcal{C})$ have been extracted from $\Omega$, the boundaries of $\Gamma_{\mathrm{h}} \backslash \Gamma_{\mathrm{c}}$ are the contours of all the $\Gamma_{\mathrm{g}}^{k}$, i.e., the contours of all the electrodes. The definition of $I_{i}$ given by (6) appears thereupon naturally, since $v_{0, s}^{i}$ is equal to 1 on the electrode contour $\gamma_{\mathrm{g}}^{i}$ and to 0 on the other electrodes contour:

$$
\int_{\partial\left(\bigcup_{k \in \mathcal{C}} \Gamma_{\mathrm{g}}^{k}\right)}\left(\mathbf{h} v_{0, s}^{i}\right) \cdot \mathrm{d} \mathbf{l}=\int_{\gamma_{\mathrm{g}}^{i}} \mathbf{h} \cdot \mathrm{d} \mathbf{l}=I_{i} .
$$

This development yields the expected circuit relation of each inductor $i$, linking its voltage $V_{i}$ and its current $I_{i}$ :

$$
\begin{aligned}
I_{i}+ & \left\langle Z_{\mathrm{c}}^{-1} \mathbf{n} \times \partial_{t} \mathbf{a}, \mathbf{n} \times \operatorname{grad} v_{0, s}^{i}\right\rangle_{\Gamma_{\mathrm{c}}^{i}} \\
& +V_{i}\left\langle Z_{\mathrm{c}}^{-1} \mathbf{n} \times \operatorname{grad} v_{0, s}^{i}, \mathbf{n} \times \operatorname{grad} v_{0, s}^{i}\right\rangle_{\Gamma_{\mathrm{c}}^{i}}=0 .
\end{aligned}
$$

\section{COMPARISON BETWEEN MASSIVE CONDUCTORS AND SIBC FORMULATIONS}

The computational gain ensuing from the use of SIBC instead of a massive conductor formulations is assessed by considering an academic example of two identical planar spiral 2 turns coils (with a $10 \mathrm{~mm}$ inner radius and made of AWG
11 wire) separated by $20 \mathrm{~mm}$. A 2D FE axisymmetric model with a highly refined mesh is used as reference. The coils spiral shape is thus approximated by concentric tori which are connected in series at the circuit level. The equivalent $3 \mathrm{D}$ system is obtained by extruding each torus section several times. The resulting geometry is meshed so that the presence of at least one element in the skin depth at the highest considered frequency is guaranteed. The problem is then solved using the massive conductors formulation [7] and the proposed SIBC formulation (with $Z_{c}$ limited to the first order) on the same mesh (the volume mesh inside the conductors being unemployed in the second case). Fig. 3 shows the relative error on the evaluation of the resistance $\mathrm{R}$ and the self-inductance $\mathrm{L}$ of one coil and the mutual inductance M between the coils.

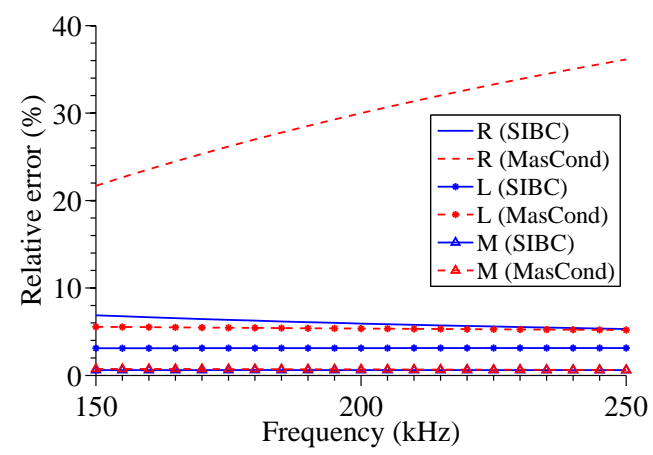

Fig. 3: Relative errors on $\mathrm{R}$ (without marker), L (dot) and $\mathrm{M}$ (triangle) with SIBC (solid) and massive conductors (dashed)

A $3.9 \mathrm{GHz}$ i7-HQ4910MQ computer with $32 \mathrm{~Gb}$ RAM is used. Each operating point includes 314381 degrees of freedom (DoF), lasting 125 seconds, using massive conductors against 142065 DoF, lasting 30 seconds, using SIBC. The computational and accuracy gains ensuing from the use of SIBC appear clearly. On a concrete example, the accuracies can be improved by using more extrusions to build the coils.

\section{EXPERIMENTAL VALIDATION}

The formulation (8)-(11) is validated experimentally on a complete WPT system depicted by the schematic on Fig. 4. The system is composed of two identical planar spiral coils, each being connected to a capacitor $C$ compensating their self-inductance in order to strengthen the performances of the transfer. As shown by preliminary tests, the capacitors present a non-negligible series resistance $R_{C}$, which has been measured and inserted in the equivalent circuit. The load is a pure resistance $R_{\mathrm{L}}$ of $3.9 \Omega$. The coils are made of 7 turns of AWG 11 wire spaced by $1.3 \mathrm{~mm}$ and present an inner radius of $80 \mathrm{~mm}$. The resonant frequency $f_{0}$ is chosen around 200 $\mathrm{kHz}$. The self-inductance of the coil is estimated analytically to be $15.7 \mu \mathrm{H}$ so that a $40 \mathrm{nF}$ capacitance is required for the capacitors. The proposed method is used for the 3D FE modeling of the coils strongly coupled with the external lumped elements. In order to lighten the geometry and the mesh of the coils, the coils are approximated by an equivalent set of 7 concentric tori connected in series. According to the selection criteria [10], the Leontovich approximation is sufficient downto $53 \mathrm{kHz}$. 


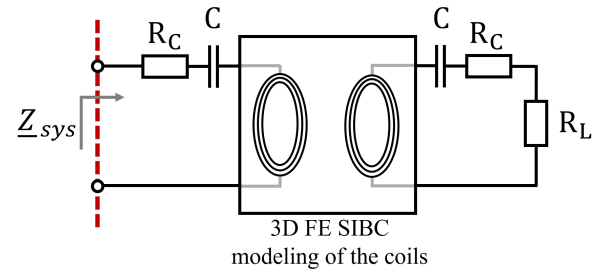

Fig. 4: Schematic of the validation example circuit

\section{A. Results when coils are aligned and separated by $10 \mathrm{~cm}$}

The input impedance $\underline{Z}_{\text {sys }}$ of the whole system when the coils are aligned and separated by $10 \mathrm{~cm}$ is determined numerically, and compared with the one measured experimentally (with a network analyzer HP 4195A combined with an impedance kit HP 41951A). Results are shown in terms of modulus $Z$ and phase $\phi_{Z}$ of $\underline{Z}_{\text {sys }}$ with respect to the operating frequency (with a focus on the resonance zone) on Fig. 5.
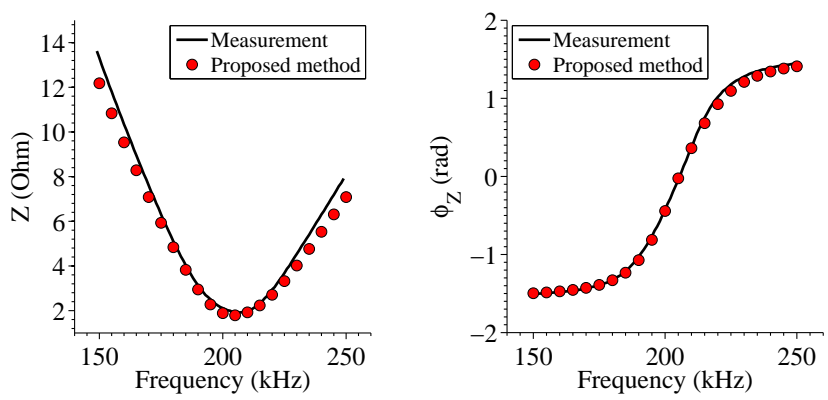

Fig. 5: Modulus (left) and phase (right) of $\underline{Z}_{\text {sys }}$ at $10 \mathrm{~cm}$ range

Each simulated frequency includes 266625 DoF, lasting 65 seconds. One can see that the simulation results and the measurements match. Slight differences are observable and are imputed to uncertainties related with the non-ideal geometry of the experimental coils, but also with the parameters of the real electrical elements surrounding the coils.

\section{B. Results when coils are aligned and separated by $5 \mathrm{~cm}$}

The same comparison is made when the coils are nearer (see Fig. 6). Each simulated frequency includes 308941 DoF, lasting 100 seconds. In this situation, the system is said to be overcoupled and presents a more complex behavior. Indeed, the system is composed by two highly coupled resonators and presents consequently two different resonances. In addition to the uncertainties mentioned in the previous section, the larger errors may be attributed to the high sensitivity of $\underline{Z}_{\text {sys }}$ to the coils coupling when the system is overcoupled. Besides, the local error around the conductors due to the use of a low order SIBC is more important as the coils are nearer. Finally, edging the coils leads logically to a larger error on $\underline{Z}_{\mathrm{sys}}$ due to the increase of the coils parasitic capacitance, which is not modeled in magnetodynamics. This last phenomenon is confirmed by the impedance patterns observed when even closer configurations are considered. However, the transfer range of applicative WPT devices has to be large, decreasing the practical impact of those low range effects. Nevertheless, our method is able to reproduce the general complex operation of two coupled resonators.
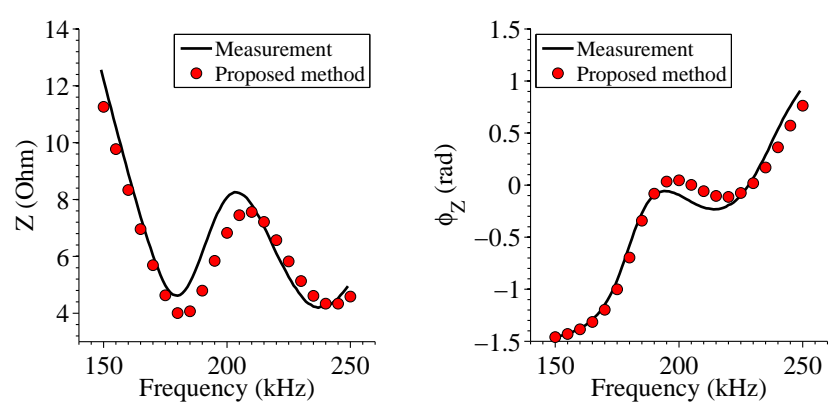

Fig. 6: Modulus (left) and phase (right) of $\underline{Z}_{\text {sys }}$ at $5 \mathrm{~cm}$ range

\section{Conclusions}

In this paper, a 3D FE formulation with SIBC and circuit coupling suitable for WPT coils modeling has been proposed. This technique enables the possibility to represent the eddy current effects while avoiding the expensive computational cost of the volume mesh inside the conductor at the concerned frequency level. Regarding the crucial aspect of the resonance in the WPT technology, a mandatory strong circuit coupling of the 3D FE $\mathbf{a}-v$ formulation involving SIBC has been developed and implemented. The computational gain ensuing from the use of SIBC has been demonstrated on an academic example. The model has been compared to experimental results and the comparison has highlighted the correct behavior of the circuit coupling while representing a WPT input impedance pattern. The observable errors are attributed to uncertainties related with the experimental circuit but also with the SIBC approximation order. The formulation being validated, the implementation of higher order SIBCs and the quantification of the ensuing impact, if any, is proposed as further work.

\section{REFERENCES}

[1] S. Li and C. Mi, "Wireless power transfer for electric vehicle applications" IEEE Trans. Emerg. Sel. Topics Power Electron., vol. 3, no. 1, pp. 4-17, Mar. 2015.

[2] A. O. Hariri, T. Youssef, A. Elsayed, and O. Mohammed, "A computational approach for a wireless power transfer link design optimization considering electromagnetic compatibility," IEEE Trans. Magn., vol. 52, no. 3, Mar. 2016.

[3] D. W. Ferreira, R. V. Sabariego, L. Lebensztajn, L. Krähenbühl, F. Morel, and C. Vollaire, "Homogenization methods in simulations of transcutaneous energy transmitters," IEEE Trans. Magn., vol. 50, no. 2, Feb. 2014.

[4] S. Yuferev, L. Di Rienzo, and N. Ida , "Surface impedance boundary conditions," ICS Newsletter, vol. 21, no. 2, pp. 3-16, Jul. 2014.

[5] S. Yuferev and L. Di Rienzo, "Surface impedance boundary conditions in terms of various formalisms," IEEE Trans. Magn., vol. 46, no. 9, pp. 3617-3628, Sep. 2010.

[6] G. Meunier, C. Guerin, and Y. Le Floch, "Circuit-Coupled t $t_{0} \phi$ formulation with surface impedance condition," IEEE Trans. Magn., vol. 44, no. 6, pp. 730-733, Jun. 2008

[7] P. Dular, F. Henrotte, and W. Legros, "A general and natural method to define circuit relations associated with magnetic vector potential formulations," IEEE Trans. Magn., vol. 35, no. 3, pp. 1630-1633, May 1999.

[8] P. Dular, A. Nicolet, A. Genon, and W. Legros, "A discrete sequence associated with mixed finite elements and its gauge condition for vector potentials," IEEE Trans. Magn., vol. 31, no. 3, pp. 1356-1359, May 1995.

[9] G. Meunier, The Finite Element Method for Electromagnetic Modeling, 1st ed., ISTE Ltd and J. Wiley and Sons, 2008.

[10] S. Yuferev and N. Ida, "Selection of the surface impedance boundary conditions for a given problem," IEEE Trans. Magn., vol. 35, no. 3, pp. 1486-1489, May 1999.

[11] A. Bossavit, "A rationale for "edge-elements" in 3-D fields computation," IEEE Trans. Magn., vol. 24, no. 1, pp. 74-79, Jan. 1988. 